\title{
UVB induces apoptosis via downregulation of CALML3-dependent JNK1/2 and ERK1/2 pathways in cataract
}

\author{
YIN JIA $^{1 *}$, QIN QIN ${ }^{1 *}$, CHAO-PING FANG $^{1}$, WEI SHEN ${ }^{2}$, TING-TING SUN ${ }^{1}$, \\ YUAN-LAN HUANG ${ }^{3}$, WEN-JIE LI $^{1}$ and AN-MEI DENG ${ }^{1}$
}

Departments of ${ }^{1}$ Laboratory Diagnosis and ${ }^{2}$ Ophthalmology, Changhai Hospital, The Second Military Medical University, Shanghai 200433; ${ }^{3}$ Medical Department, The 455th Hospital of PLA, Shanghai 200052, P.R. China

Received November 13, 2015; Accepted January 19, 2018

DOI: $10.3892 /$ ijmm.2018.3478

\begin{abstract}
The aim of the current study was to understand the mechanisms of apoptosis occurring in cultured human lens epithelial cells (HLECs) following ultraviolet B (UVB) irradiation. The investigations intended to confirm the presence of apoptosis and to reveal the roles of oxidative stress, calcium $\left(\mathrm{Ca}^{2+}\right)$, c-Jun NH2-terminal kinase (JNK)1/2, and extracellular signal-regulated kinase (ERK)1/2 signaling pathway in these progresses. Cell apoptosis, ROS generation and intracellular $\mathrm{Ca}^{2+}$ concentration was measured by flow cytometry. The expression of CALML3, caspase-3, Bax, Bcl-2, p-JNK1/2, JNK1/2, p-ERK1/2 and ERK1/2 was measured by RT-qPCR and western blot analysis. Annexin V-fluorescein isothiocyanate/propidium iodide staining demonstrated that UVB irradiation increased the apoptotic rate, reactive oxygen species (ROS) production and intracellular $\mathrm{Ca}^{2+}$ concentration of HLECs in dose- and time-dependent manners. Overexpression of calmodulin like 3 (CALML3) reversed the effects of UVB irradiation on apoptosis, ROS production and $\mathrm{Ca}^{2+}$ concentration of HLECs, and decreased expressions of caspase- 3 and Bax, with increased expressions of Bcl-2. Notably, silencing of CALML3 had similar effects to UVB irradiation and inhibited the activation JNK1/2 and ERK1/2 pathways. Nimodipine, a $\mathrm{Ca}^{2+}$-channel antagonist, significantly attenuated the damages induced by CALML3 downregulation. In conclusion, UVB irradiation induced increase in apoptosis, $\mathrm{ROS}$ production and $\mathrm{Ca}^{2+}$ concentration of HLECs, in part, by downregulating the expression
\end{abstract}

Correspondence to: Dr Wen-Jie Li or Dr An-Mei Deng, Department of Laboratory Diagnosis, Changhai Hospital, The Second Military Medical University, 168 Changhai Road, Yangpu, Shanghai 200433, P.R. China

E-mail: liwenjie_lwj@126.com

E-mail: amdeng70@163.com

*Contributed equally

Key words: cataract, ultraviolet B irradiation, human lens epithelial cells, calmodulin like 3, apoptosis, c-Jun NH2-terminal kinase 1/2, extracellular signal-regulated kinase $1 / 2$ of CALML3 and involved oxidative stress, $\mathrm{Ca}^{2+}, \mathrm{JNK} 1 / 2$ and ERK1/2 signaling pathways, suggesting that investigating CALML3 may useful for developing cataract treatment.

\section{Introduction}

Cataracts are a cause major cause of blindness worldwide. It is reported that a number of factor lead to the development and progression of cataracts, including $\mathrm{H}_{2} \mathrm{O}_{2}$, glucose and prolonged exposure to sunlight $(1,2)$. Ultraviolet $\mathrm{B}$ (UVB) is associated with the formation of cataracts leading to cortical lens opacity. The lens epithelium is a single layer of cells that regulates lens homeostasis; however, the lens epithelium is exposed to UVB irradiation, as the top layer of cells in the eye (3). Even relatively low UVB irradiation damages the lens epithelium and causes a loss of ion homeostasis, which contributes to the development of cataracts in the aging population (4). The precise mechanism of the association of UVB irradiation with cataract development in humans unknown.

Apoptosis is a regulated cell death mechanism that maintains homeostasis, and has a role in cataract formation in humans (5). Loss of mitochondrial transmembrane potential and the production of reactive oxygen species (ROS) cause damage to cells and induce apoptosis. UVB irradiation induces apoptosis through a number of molecular processes, such as targeting Bcl-2 family proteins, and activating caspase and cell apoptosis signaling pathways (6). Bcl-2 has a negative role in the cellular apoptotic pathway, whereas Bax, a Bcl-2 homologue, can reverse the inhibitory effect of Bcl-2 in apoptosis. A previous study demonstrated that Bax expression and caspase-3 activity were decreased in cataracts, suggesting a pivotal role in cataract formation (7). The mitogen-activated protein kinases (MAPKs), including extracellular signal-regulated kinase (ERK), c-Jun NH2-terminal kinase (JNK) and p38 MAPK, are involved in the regulation of cell apoptosis and are activated during UVB-induced apoptosis of human retinal pigment epithelial cells $(8,9)$.

Oxidative stress caused by ROS has been reported in numerous diseases, particularly aged-associated disorders, such as cataracts (10). Previous studies demonstrated that the lens has an antioxidant system to counter the toxic damage of ROS, including the activity of antioxidantive enzymes and antioxidants, such as superoxide dismutase (SOD), catalase 
(CAT), glutathione peroxidase (GSH-PX) and malondialdehyde (MDA) (11-13). Chang et al (14) reported that the serum SOD, CAT and GSH-PX activities were decreased and the level of MDA was increased in patients with cataracts.

Cellular calcium $\left(\mathrm{Ca}^{2+}\right)$ homeostasis in the lens epithelial cells is essential for maintaining lens clarity. Inappropriate $\mathrm{Ca}^{2+}$ signaling and abnormal $\mathrm{Ca}^{2+}$ levels are associated with various clinical disorders, including stroke, cardiac dysfunction, obesity, aging and Alzheimer's diseases (15-18). $\mathrm{Ca}^{2+}$ is also involved in cell growth, differentiation and motility during the transformation and progression of cancer. Calmodulin like 3 (CALML3) is an epithelial-specific calcium sensing protein that highly expressed in oral mucosa, skin, breast and prostate $(19,20)$. A previous study reported that CALML3 is regulated by short-term blockade of the MaxiK channel associated with an increase in basal $\mathrm{Ca}^{2+}$ concentration (21). However, the function of CALML3 in cataracts has not been fully elucidated and requires further investigation.

The current study investigated the effect of UVB irradiation on apoptosis, and whether oxidative stress, $\mathrm{Ca}^{2+}$ homeostasis and apoptosis-associated signaling pathways have a role in UVB-induced apoptosis in human lens epithelium cells.

\section{Materials and methods}

Cell culture. Human lens epithelial cells (HLEC; an immortalized cell line) used in this study obtained from Cell Bank of Academia Sinica (Shanghai,China), were cultured in Dulbecco's modified Eagle's medium (DMEM)-high glucose (HyClone; GE Healthcare Life Sciences, Logan, UT, USA) supplemented with $10 \%$ fetal bovine serum (FBS) and $1 \%$ penicillin-streptomycin solution in an incubator (Thermo Fisher Scientific, Inc., Waltham, MA, USA) at $37^{\circ} \mathrm{C}$ with $5 \% \mathrm{CO}_{2}$. Cells were treated with $0.04 \mathrm{mM}$ nimodipine for $24 \mathrm{~h}$

UVB treatment. The UVB treatment was performed by using a UVB radiometer with a total output of $4 \mathrm{~W} / \mathrm{m}^{2}$ (wavelengths of $297 \mathrm{~nm}$ ). Prior to irradiation, the HLECs were washed twice with phosphate-buffered saline (PBS). The HLECs were then irradiated at the desired intensity and time: $60 \mathrm{~min}$ at $0.5,1$, 2 and $4 \mathrm{~W} / \mathrm{m}^{2} ; 2 \mathrm{~W} / \mathrm{m}^{2}$ for $5,15,30,60,90,120$ and $180 \mathrm{~min}$. Following UVB irradiation the HLECs were incubated in DMEM-high glucose (HyClone; GE Healthcare Life Sciences) supplemented with $10 \%$ FBS and $1 \%$ penicillin-streptomycin solution in an incubator (Thermo Fisher Scientific, Inc.) at $37^{\circ} \mathrm{C}$ with $5 \% \mathrm{CO}_{2}$.

Lentivirus and small interfering RNA transduction. The CALML3 coding sequence (GENEWIZ lnc., Suzhou, China) was cloned into the pLVX-AcGFP-C1 lentivirus vector (Addgene, Inc., Cambridge, MA, USA). Lipofectamine ${ }^{\circledR} 2000$ (Invitrogen; Thermo Fisher Scientific, Inc.) was used to transfect small interfering RNA (siRNA; $40 \mathrm{nM}$; 5'-AUCUCUCUGUUGCUACUCA-3') targeting human CALML3 mRNA (position 1288-1310 5'-ACCUGUUCCUAUUCAUCUG-3') and constructs according to the manufacture's instruction. Empty lentivirus vector and nonspecific siRNA were used as the negative control (NC). Cells were analyzed at $48 \mathrm{~h}$ after transduction and transfection.
Flow cytometry analysis of apoptosis, ROS production and calcium concentration. Prior to analysis of analysis of apoptosis, ROS production and calcium concentration, the HLECs were trypsinized, centrifuged and resuspended twice with PBS. The HLECs $\left(1 \times 10^{5} \mathrm{cell} / \mathrm{ml}\right)$ were treated with UVB with $2 \mathrm{~W} / \mathrm{m}^{2}$ for $60 \mathrm{~min}$, and subsequently collected and incubated with $5 \mu \mathrm{l}$ Annexin $\mathrm{V}$ buffer and $5 \mu \mathrm{l}$ propidium iodide (PI) for $10 \mathrm{~min}$ for apoptosis detection, incubated with medium containing $10 \mu \mathrm{M}$ dichlorodihydrofluorescein diacetate (DCFH-DA) for $30 \mathrm{~min}$ for ROS detection and incubated with Fluo-3 $(10 \mu \mathrm{M}$; Sigma-Aldrich; Merck KGaA, Darmstadt, Germany) for 30 min for intracellular calcium concentration detection.

Determination of SOD, MDA, CAT and GSH-PX activities. The HLECs $\left(1 \times 10^{5} \mathrm{cell} / \mathrm{ml}\right)$ were treated with UVB with $2 \mathrm{~W} / \mathrm{m}^{2}$ for $60 \mathrm{~min}$. The SOD (WST-1 method), MDA (TBA method), CAT (visible light) and GSH-PHX assay kits (obtained from Nanjing Jiancheng Bioengineering Institute, Nanjing, China) were used to determine the antioxidant activities. SOD, MDA, CAT and GSH-PHX activities were measured at the absorbance of 450, 532, 405 and $412 \mathrm{~nm}$, respectively.

Reverse transcription-quantitative polymerase chain reaction (RT-qPCR) assay. Total RNA was isolated with TRIzol reagent (Invitrogen; Thermo Fisher Scientific, Inc.) according to the manufacturer's protocols. Complementary DNA was synthesized using a cDNA synthesis kit (Thermo Fisher Scientific, Inc.). The cDNA synthesis conditions were as follows: $37^{\circ} \mathrm{C}$ for $60 \mathrm{~min}$, followed by $85^{\circ} \mathrm{C}$ for $5 \mathrm{~min}$ and $4^{\circ} \mathrm{C}$ for 5 min. qPCR was performed to detect the mRNA levels of the indicated genes. qPCR was performed on ABI 7300 (Applied Biosystems; Thermo Fisher Scientific, Inc.) thermal cycler using a standard SYBR-Green PCR kit (Thermo Fisher Scientific, Inc.) according to the manufacturer's instructions. The qPCR cycling conditions were as follows: $95^{\circ} \mathrm{C}$ for $10 \mathrm{~min}$, followed by 40 cycles at $95^{\circ} \mathrm{C}$ for $15 \mathrm{sec}$ and $60^{\circ} \mathrm{C}$ for $45 \mathrm{sec}$, and a final extension step of $95^{\circ} \mathrm{C}$ for $15 \mathrm{sec}, 60^{\circ} \mathrm{C}$ for $1 \mathrm{~min}, 95^{\circ} \mathrm{C}$ for $15 \mathrm{sec}$ and $60^{\circ} \mathrm{C}$ for $15 \mathrm{sec}$. The relative mRNA expression of target gene compared with GAPDH was calculated using the $2^{-\Delta \Delta C q}(22)$ method. The primers used in this study listed in Table I.

Western blot assay. HLECs were seeded at a density of $5 \times 10^{5}$ cells per well in 6-well plates, cultured overnight and then treated with UVB $2 \mathrm{~W} / \mathrm{m}^{2}$ for 60 and $180 \mathrm{~min}$. Total proteins were isolated from the HLECs and were subjected to $12 \%$ SDS-PAGE, electroblotted onto to a polyvinylidene fluoride membrane (Roche Diagnostics, $\mathrm{GmbH}$, Mannheim, Germany). Following blocking with 5\% skimmed milk at $4^{\circ} \mathrm{C}$ overnight, membranes were first incubated with mouse polyclonal antibody anti-CALML3 (cat. no. ab88761; 1:1,000); rabbit monoclonal antibodies anti-caspase-3 (cat. no. ab32351; 1:5,000), anti-Bax (cat. no. ab32503; 1:1,000; all from Abcam, Cambridge, MA, USA), anti-ERK1/2 (cat. no. 4695; 1:1,000), anti-phospho-ERK1/2 (cat. no. 4376; 1:800) and anti-GAPDH (cat. no. 5174; 1:1500; all from Cell Signaling Technology, Inc., Danvers, MA, USA); mouse monoclonal antibodies anti-Bcl-2 (cat. no. ab117115; 1:1,000; Abcam) and antibodies anti-phospho-JNK1/2 (cat. no. 9255; 1:1,000); rabbit polyclonal antibodies anti-JNK1/2 (cat. no. 9252; 1:1,000; both 
A

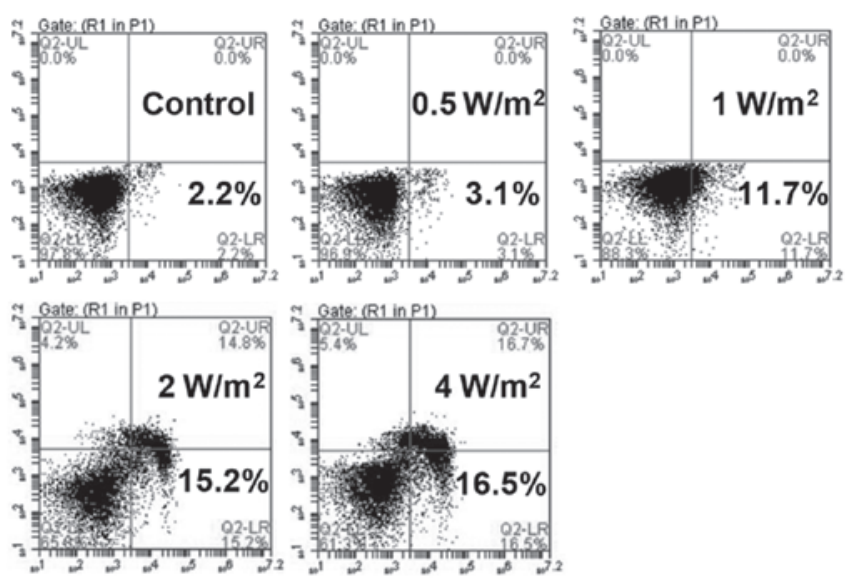

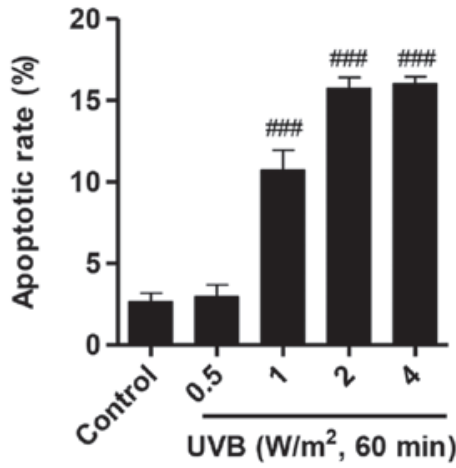

B
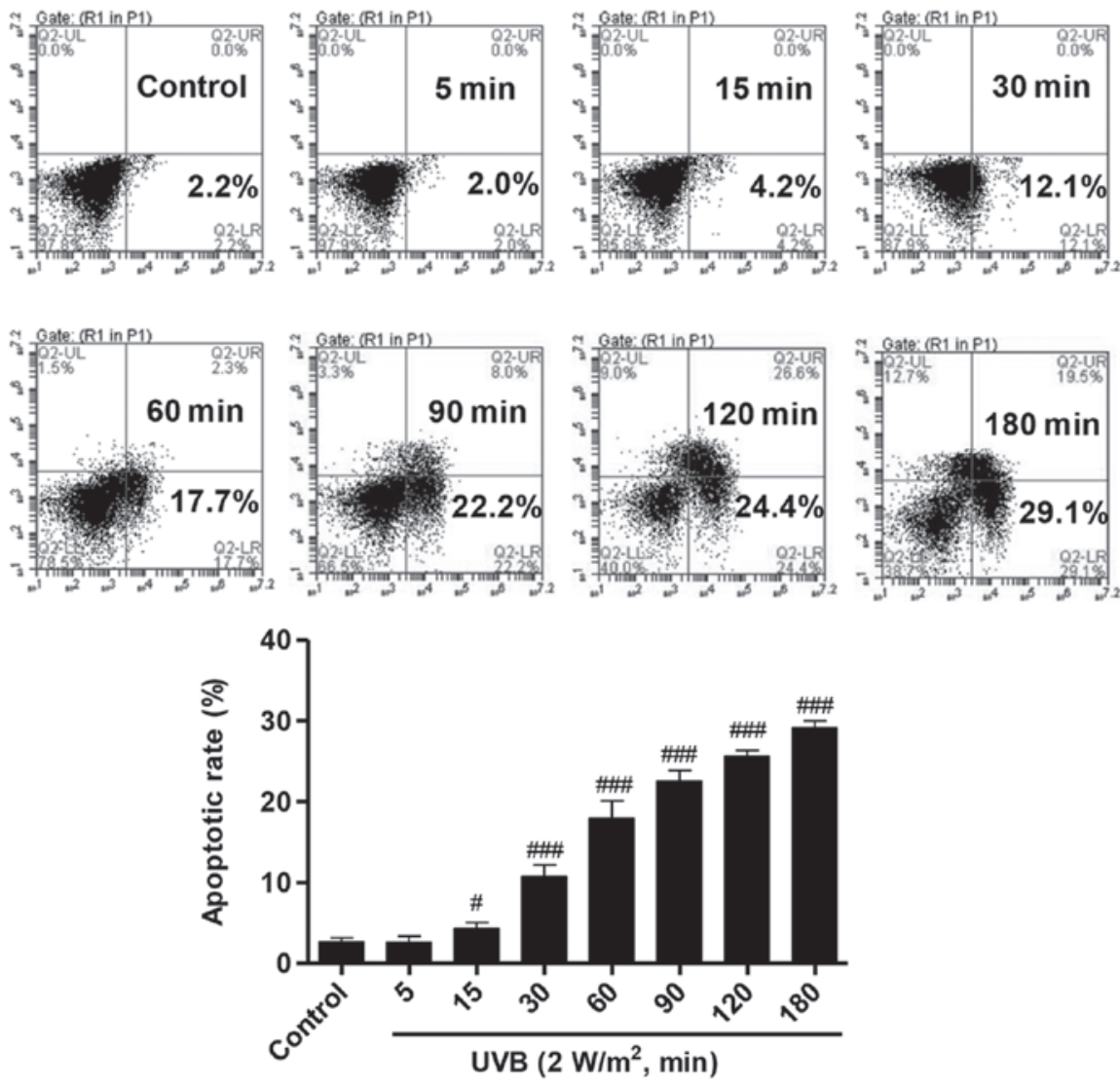

Figure 1. UVB induces apoptosis of cultured HLECs. (A) HLECs were exposed to different intensities of UVB $\left(0.5,1,2\right.$ and $\left.4 \mathrm{~W} / \mathrm{m}^{2}\right)$ for $60 \mathrm{~min}$. (B) HLECs were exposed to $2 \mathrm{~W} / \mathrm{m}^{2} \mathrm{UVB}$ for different times $(5,15,30,60,90,120$ and $180 \mathrm{~min})$. Cell apoptosis was determined by flow cytometry. ${ }^{\# \# ~} \mathrm{P}<0.001 \mathrm{vs}$. control. UVB, ultraviolet B; HLECs, human lens epithelial cells.

from Cell Signaling Technology, Inc.). Blots were then incubated with goat anti-mouse or anti-rabbit secondary antibody (cat. nos. A0208 and A0216; 1:1,000; Beyotime Institute of Biotechnology, Haimen, China) and visualized using enhanced chemiluminescence (Thermo Fisher Scientific, Inc.). GAPDH antibody was used as an internal control. The blotting bands were quantified with ImageJ software (National Institutes of Health, Bethesda, MD, USA).

Statistical analysis. The data was presented as the mean value + standard deviation. Data were analysed by one-way analysis of variance followed by Tukey's post hoc test. Statistical analyses were performed using GraphPad Prism 5 software (GraphPad Software, Inc., La Jolla, CA, USA). P<0.05 was considered to indicate a statistically significant difference.

\section{Results}

$U V B$ induces apoptosis of HLECs. To investigate the effects of UVB irradiation on HLECs, the HLECs were treated with several of doses of UVB $\left(0.5,1,2\right.$ and $\left.4 \mathrm{~W} / \mathrm{m}^{2}\right)$ for $60 \mathrm{~min}$. Apoptosis analysis was performed following UVB irradiation using Annexin V/PI labeling followed by flow cytometry. The percentage of apoptotic cells in HLECs with different doses of UVB irradiation was significantly increased compared with that of the control group without UVB irradiation (Fig. 1A). 

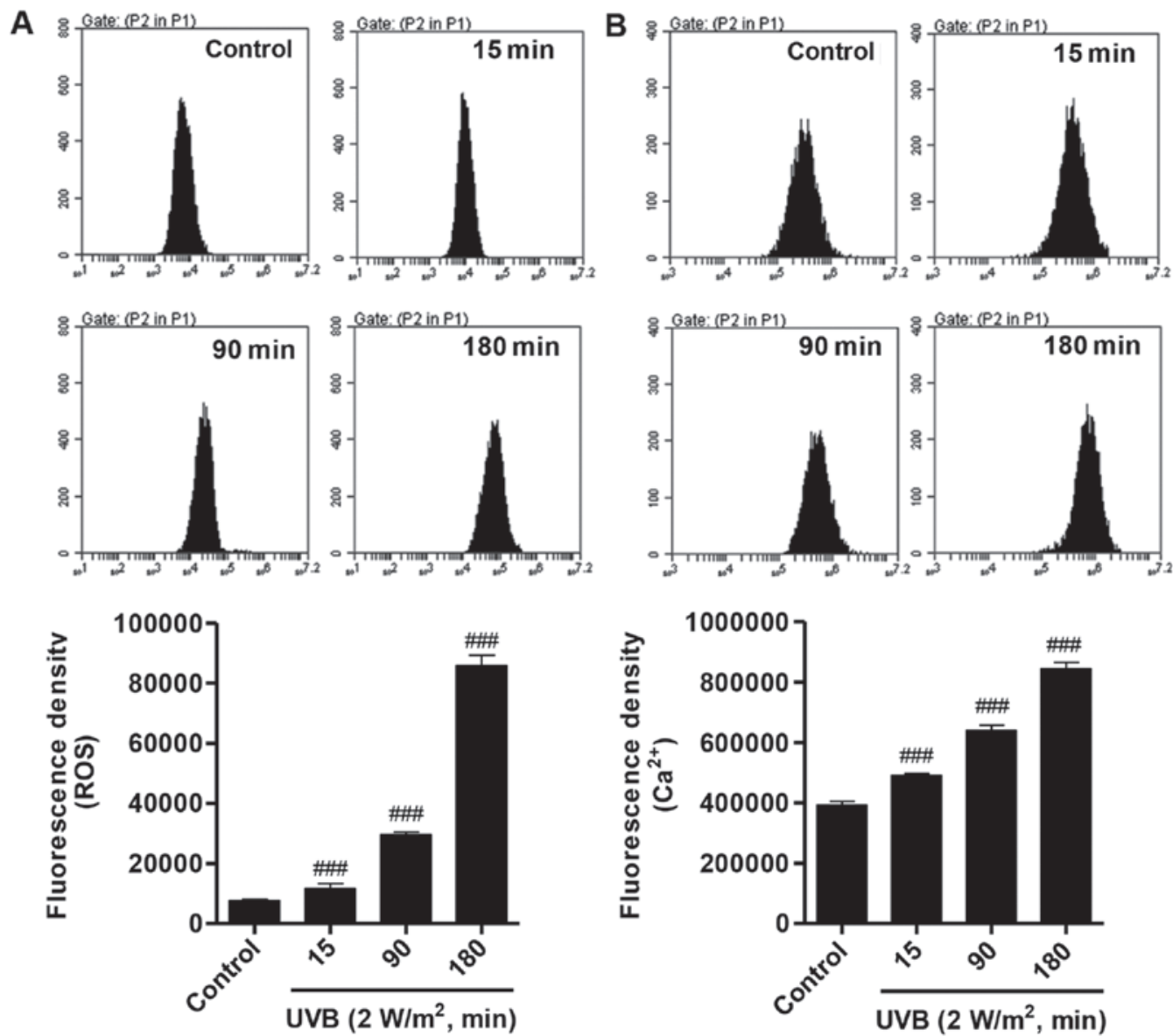

Figure 2. UVB induces ROS production and intracellular $\mathrm{Ca}^{2+}$ concentration increase. HLECs were exposed to $2 \mathrm{~W} / \mathrm{m}^{2} \mathrm{UVB}$ for different times $(15,90$ and $180 \mathrm{~min}$ ). (A) Following UVB irradiation, the ROS production of HLECs was significantly increased in a time-dependent manner. (B) Following UVB irradiation, the intracellular $\mathrm{Ca}^{2+}$ concentration of HLECs was significantly increased in a time-dependent manner. ${ }^{\# \# \prime} \mathrm{P}<0.001$ vs. control. UVB, ultraviolet $\mathrm{B}$; ROS, reactive oxygen species; HLECs, human lens epithelial cells.

There was no significant difference in the number of apoptotic between the groups treated with $2 \mathrm{~W} / \mathrm{m}^{2}$ and $4 \mathrm{~W} / \mathrm{m}^{2} \mathrm{UVB}$ irradiation, thus $2 \mathrm{~W} / \mathrm{m}^{2} \mathrm{UVB}$ irradiation was used in subsequent experiments. To further determine the effects of UVB irradiation on apoptosis of HLECs, the cells were also treated with $2 \mathrm{~W} / \mathrm{m}^{2} \mathrm{UVB}$ for various durations $(5,15,30,60,90,120$ and $180 \mathrm{~min}$ ). After $15 \mathrm{~min}$ irradiation, the number of apoptotic cells $\sim 4.2 \%$, and the percent of apoptotic cells was increased with the increasing duration of UVB irradiation (Fig. 1B). These results observed that UVB irradiation induces apoptosis in the HLECs in dose- and time-dependent manners.

UVB increases ROS production and intracellular $\mathrm{Ca}^{2+}$ concentration. A number of studies have reported that oxidative stress is associated with the cell damage that occurs during exposure to UV irradiation. Whether UVB irradiation can affect the ROS production and intracellular $\mathrm{Ca}^{2+}$ concentration was investigated. DCFH-DA is a dye that reacts with ROS used for the detection of ROS level. The results demonstrated that ROS was notably increased following $2 \mathrm{~W} / \mathrm{m}^{2}$ UVB irradiation in a time-dependent manner, compared with the control group (Fig. 2A). Similarly, following $2 \mathrm{~W} / \mathrm{m}^{2} \mathrm{UVB}$ irradiation, the intracellular $\mathrm{Ca}^{2+}$ concentration was increased in a time-dependent manner (Fig. 2B). These data indicated that UVB irradiation increases oxidative stress in HLECs.
Effect of $U V B$ on CALML3 expression. To further examine the effect of UVB irradiation on calcium metabolism, CALML3 expression was determined using RT-qPCR and western blot analysis. Following treatment with $2 \mathrm{~W} / \mathrm{m}^{2} \mathrm{UVB}$ for 15,90 and $180 \mathrm{~min}$, CALML3 was significantly decreased at the mRNA and protein levels (Fig. 3). These results suggest that the pro-apoptotic activity of UVB may be associated with downregulating CALML3 expression.

Overexpression of CALML3 reduces apoptosis, ROS production and intracellular $\mathrm{Ca}^{2+}$ concentration. To elucidate whether the pro-apoptotic activity of UVB irradiation was associated with the downregulation of CALML3 expression in HLECs, a CALML3 overexpression vector was introduced into HLECs. The expression of CALML3 in the overexpression group was increased compared with the NC group, measured by western blot analysis (Fig. 4A). In HLECs treated with $2 \mathrm{~W} / \mathrm{m}^{2} \mathrm{UVB}$ irradiation for $90 \mathrm{~min}$, the percentage of apoptotic cells in CALML3 overexpression group was significantly reduced compared with the NC group (Fig. 4B). Furthermore, flow cytometry revealed that the fluorescence densities of ROS and $\mathrm{Ca}^{2+}$ were significantly reduced in CALML3-overexpressed HLECs following UVB irradiation (Fig. 4C and D). These results demonstrated that the apoptotic effect of UVB may mainly through downregulation of CALML3. 
Table I. Primers sequences used in this study.

\begin{tabular}{lrr}
\hline Gene & \multicolumn{1}{c}{ Forward } & Reverse \\
\hline CALML3 & 5'-GGCCAGGTCAATTATGAAG-3' & 5'-TCAGGGAAGAAGGAGAAAG-3' \\
Caspase-3 & 5'-AACTGGACTGTGGCATTGAG-3' & 5'-ACAAAGCGACTGGATGAACC-3' \\
Bax & 5'-AGCTGAGCGAGTGTCTCAAG-3' & 5'-TGTCCAGCCCATGATGGTTC-3' \\
Bcl-2 & 5'-AGACCGAAGTCCGCAGAACC-3' & 5'-GAGACCACACTGCCCTGTTG-3' \\
GAPDH & 5'-CACCCACTCCTCCACCTTTG-3' & 5'-CCACCACCCTGTTGCTGTAG-3'
\end{tabular}

CALML3, calmodulin like 3; GAPDH, glyceraldehyde 3-phosphate dehydrogenase.
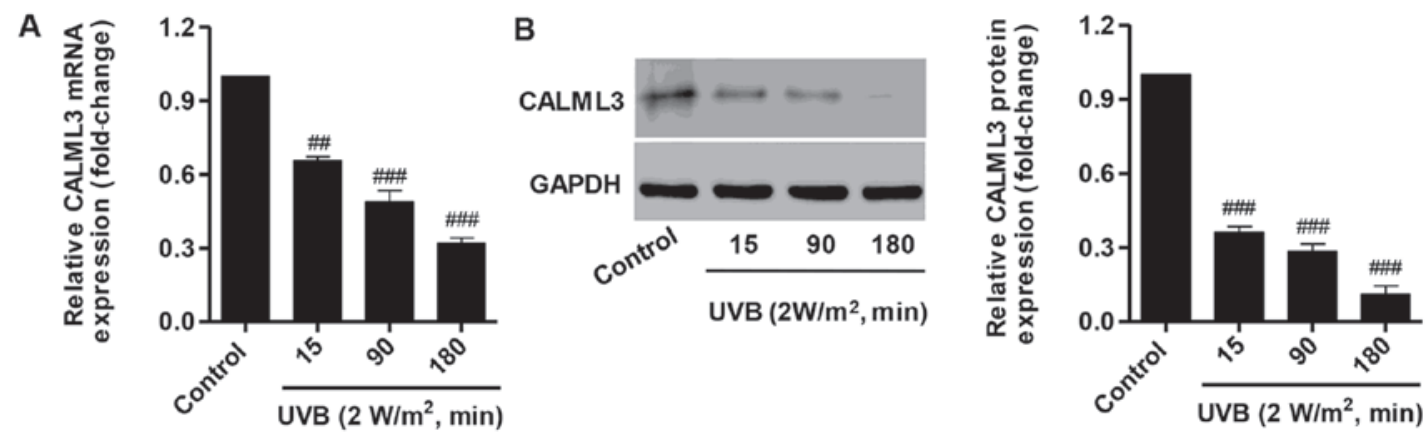

Figure 3. UVB irradiation downregulates CALML3 expression. Following $2 \mathrm{~W} / \mathrm{m}^{2} \mathrm{UVB}$ irradiation for different times (15, 90 and $\left.180 \mathrm{~min}\right)$, the expression levels of human lens epithelial cells were significantly decreased in a time-dependent manner in (A) mRNA and (B) protein. ${ }^{\# \#} \mathrm{P}<0.01$ and ${ }^{\# \# \#} \mathrm{P}<0.001$ vs. control. CALML3, calmodulin like 3; UVB, ultraviolet B.

A

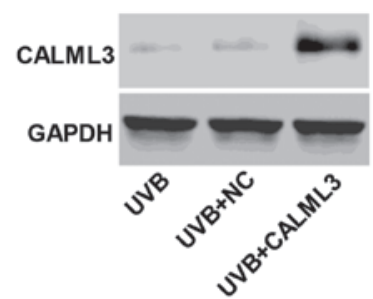

B
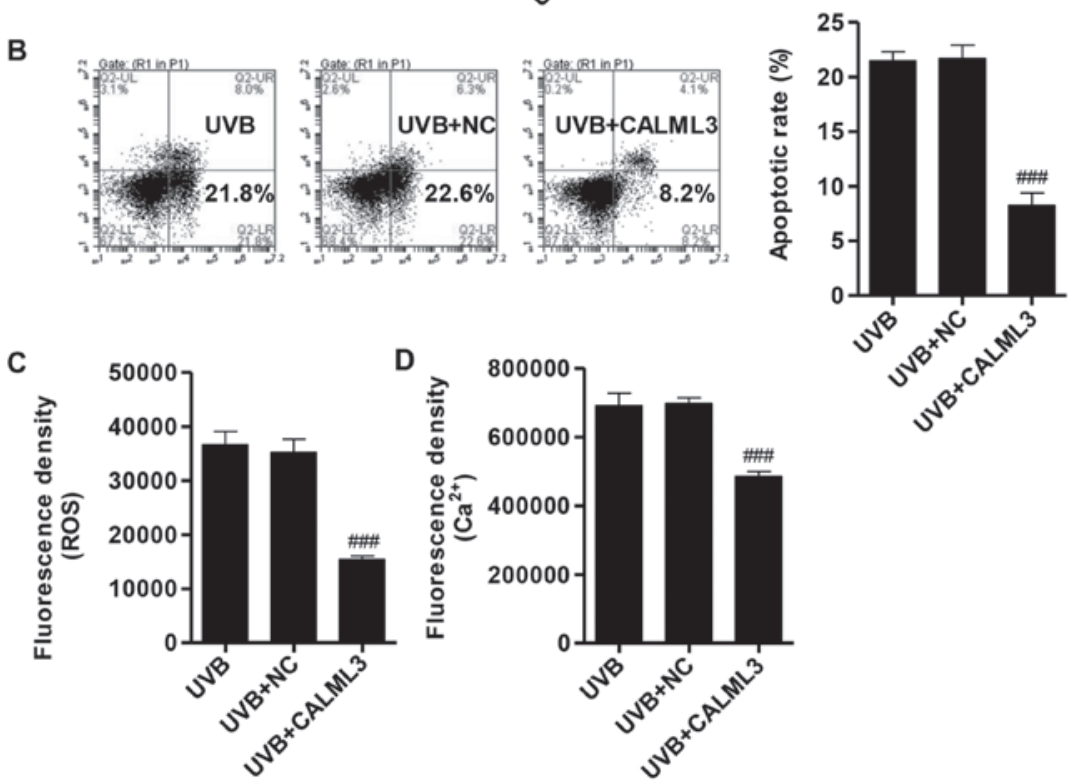

Figure 4. CALML3 overexpression decreases UVB-induced apoptosis, ROS production and intracellular $\mathrm{Ca}^{2+}$ concentration in HLECs. HLECs were exposed to $2 \mathrm{~W} / \mathrm{m}^{2} \mathrm{UVB}$ for $90 \mathrm{~min}$. (A) The protein levels of CALML3 in UVB-induced HLECs with CALML3 overexpression. Overexpression of CALML3 was significantly decreased the (B) apoptosis, (C) ROS production and (D) intracellular $\mathrm{Ca}^{2+}$ concentration in UVB-induced HLECs. ${ }^{\# \#} \mathrm{P}<0.001 \mathrm{vs.} \mathrm{UVB.}$ CALML3, calmodulin like 3; UVB, ultraviolet B; NC, negative control; ROS, reactive oxygen species; HLECs, human lens epithelial cells. 

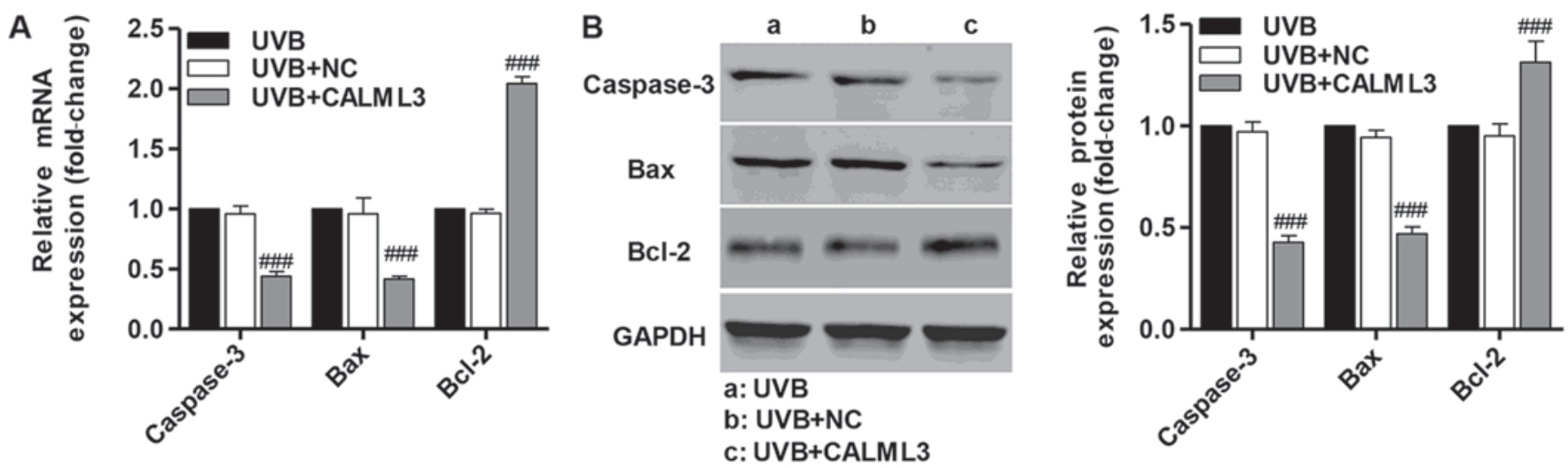

Figure 5. Effect of CALML3 overexpression on apoptosis associated proteins in UVB-induced HLECs. HLECs were exposed to $2 \mathrm{~W} / \mathrm{m}^{2} \mathrm{UVB}$ for $90 \mathrm{~min}$. The expression level of caspase-3 and Bax/Bcl-2 ratio were significantly decreased in UVB-induced HLECs with CALML3 overexpression in both (A) mRNA and (B) protein. ${ }^{\# \# ~} \mathrm{P}<0.001$ vs. UVB. CALML3, calmodulin like 3; UVB, ultraviolet B; NC, negative control; HLECs, human lens epithelial cells.
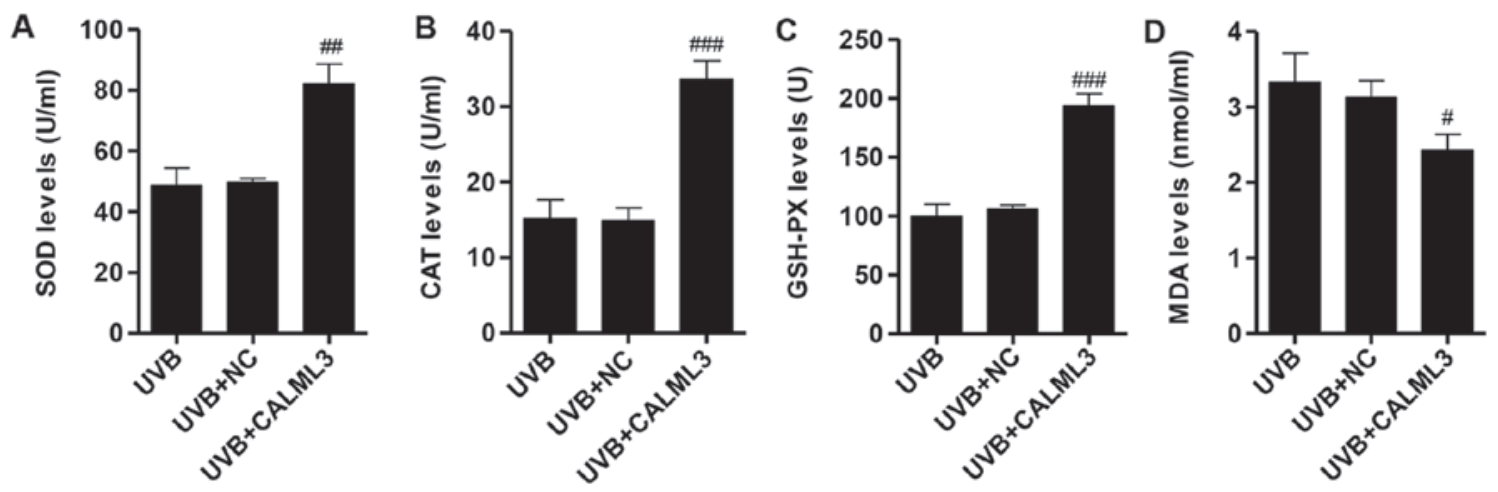

Figure 6. Evaluation of oxidative stress after exposure with UVB irradiation in CALML3-overexpressed HLECs. The levels of (A) SOD, (B) CAT and (C) GSH-PX were significantly increased in UVB-induced HLECs with CALML3 overexpression. (D) MDA was significantly decreased in UVB-induced HLECs with CALML3 overexpression. ${ }^{\#} \mathrm{P}<0.05,{ }^{\# \#} \mathrm{P}<0.01$ and ${ }^{\# \# /} \mathrm{P}<0.001$ vs. UVB. SOD, superoxide dismutase; CAT, catalase; GSH-PX, glutathione peroxidase; MDA, malondialdehyde; CALML3, calmodulin like 3; UVB, ultraviolet B; NC, negative control; HLECs, human lens epithelial cells.

Overexpression of CALML3 alters the expression of apoptosis-associated proteins. RT-qPCR and western blot analysis were performed following UVB treatment for $180 \mathrm{~min}$ to detect caspase-3, Bax and Bcl-2 in UVB-induced HLECs in the presence of CALML3 overexpression. CALML3 overexpression markedly increased the expression of the anti-apoptotic protein $\mathrm{Bcl}-2$, and reduced the expression of the pro-apoptotic proteins caspase- 3 and Bax compared with the negative control UVB-induced HLECs without CALML3 overexpression (Fig. 5). These findings demonstrate that CALML4 regulates the expression of apoptosis-associated proteins and may be involved in UVB-induced HLEC apoptosis.

Measurement of SOD, MDA, CAT and GSH-PX levels. To determine potential changes of SOD, MDA, CAT and GSH-PX in HLECs upon UVB irradiation, biochemistry assay kits were used to measure the oxidative stress-induced alteration in HLECs. Compared with the NC group with $2 \mathrm{~W} / \mathrm{m}^{2} \mathrm{UVB}$ irradiation for $90 \mathrm{~min}$, the SOD, CAT and GSH-PX levels of HLECs were significantly increased by CALML3 overexpression (Fig. 6A-C). Contrary to the increased SOD, CAT and GSH-PX levels, the MDA level of HLECs in the presence of UVB irradiation was decreased by
CALML3 overexpression (Fig. 6D). These results indicated that CALML3 overexpression can reverse the UVB-induced oxidative stress in HLECs.

Silencing of CALML3 increases apoptosis, ROS production and intracellular $\mathrm{Ca}^{2+}$ concentration. To elucidate whether CALML3 downregulation has similar effects to UVB irradiation, CALML3 siRNA (siCALML3) was transfected into HLECs and the expression of CALML3 was significant decreased by the siRNA compared with NC group, as measured by RT-qPCR (Fig. 7A). The number of apoptotic HLECs was significantly increased by CALML3 downregulation compared with $\mathrm{NC}$, however, when combined with nimodipine, a $\mathrm{Ca}^{2+}$-channel antagonist, apoptosis was significantly decreased compared with siCALML3 (Fig. 7B). The fluorescence densities of ROS and $\mathrm{Ca}^{2+}$ in HLECs were significantly increased by siCALML3 compared with NC, and nimodipine decreased the levels of ROS and $\mathrm{Ca}^{2+}$ compared with the siCALML3 group (Fig. 7C and D). Nimodipine treatment significantly reduced the modulations in apoptosis, ROS production, and intracellular $\mathrm{Ca}^{2+}$ concentration induced by siCALML4 treatment alone, (Fig. 7B-D). These results suggest that the calcium signaling pathway is associated with the CALML3-induced apoptosis in HLECs. 


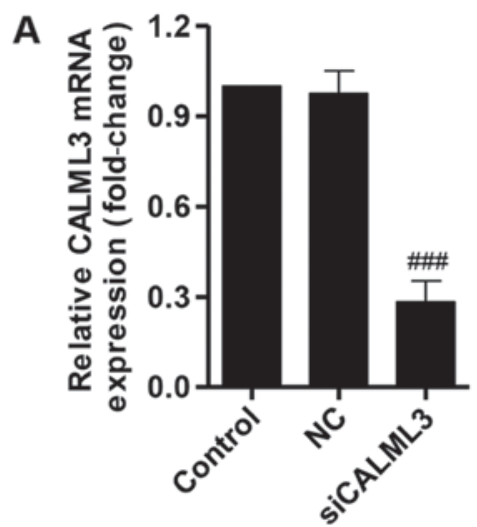

B

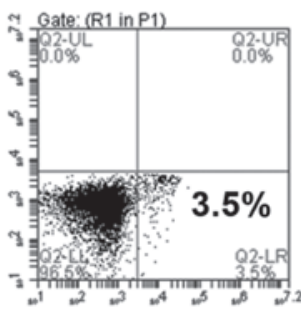

NC

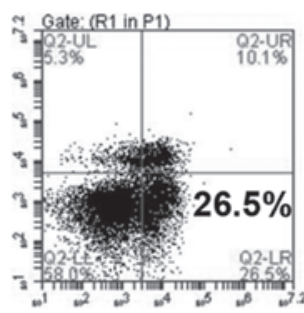

SICALML3

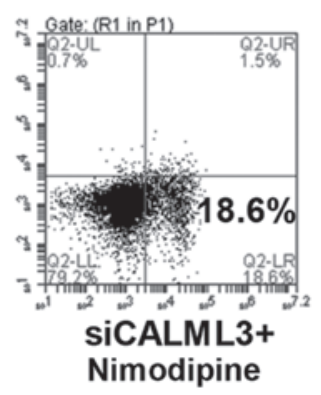

Nimodipine

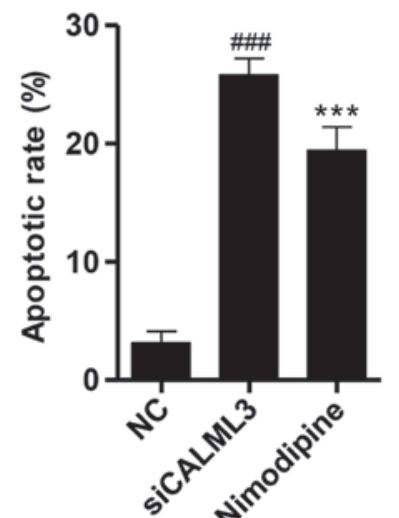

C
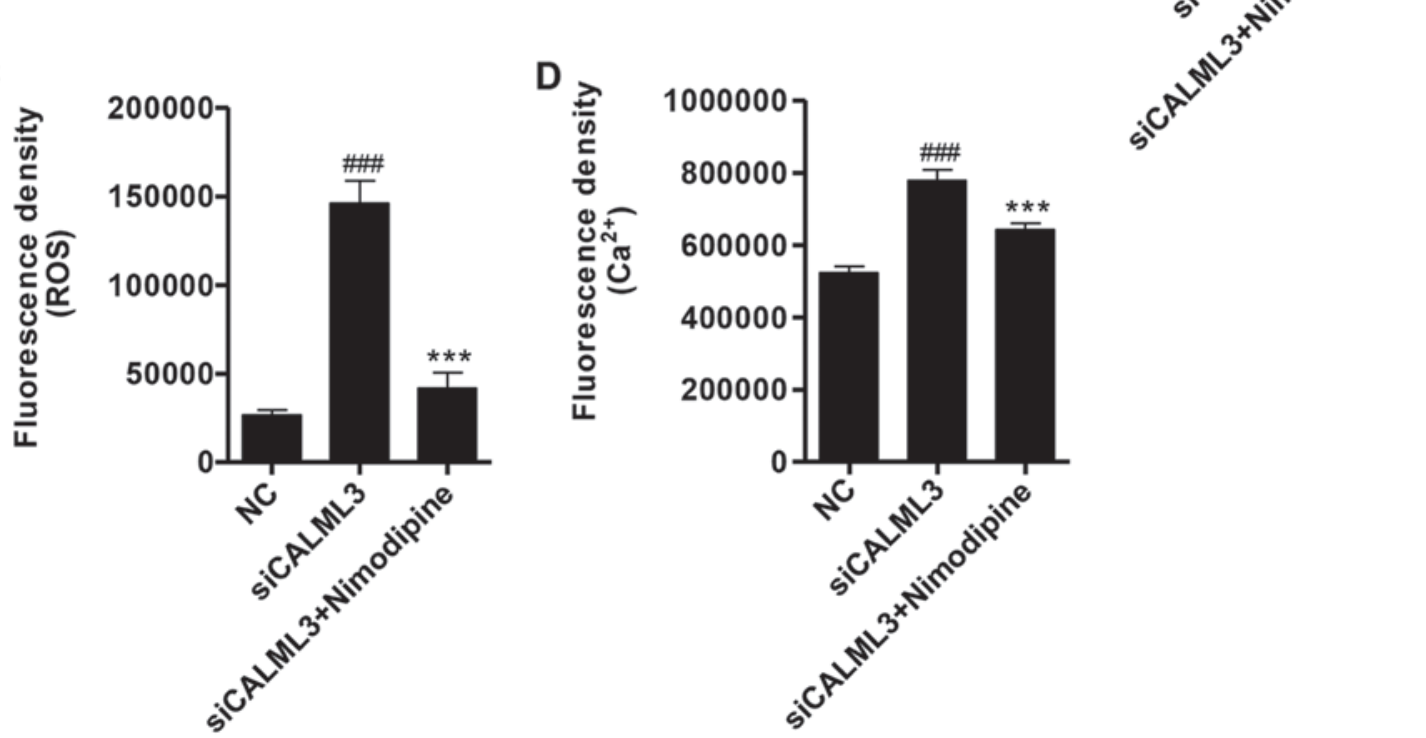

Figure 7. Silencing of CALML3 induces apoptosis, ROS production and intracellular $\mathrm{Ca}^{2+}$ concentration increase in HLECs. (A) The mRNA levels of CALML3 in HLECs with siCALML3 transfection. (B) Silencing of CALML3 was significantly increased the apoptosis, (C) ROS production and (D) intracellular Ca ${ }^{2+}$ concentration in HLECs without ultraviolet B irradiation. However, nimodipine treatment inhibited the increase in apoptosis, ROS production and intracellular $\mathrm{Ca}^{2+}$ concentration induced by siCALML3 in HLECs. ${ }^{\# \# t} \mathrm{P}<0.001$ vs. $\mathrm{NC}$ and ${ }^{* * * *} \mathrm{P}<0.001$ vs. siCALML3. CALML3, calmodulin like 3; NC, negative control; siCALML3, CALML3 small interfering RNA; ROS, reactive oxygen species; HLECs, human lens epithelial cells.

Effect of CALML3 silencing in apoptosis-associated proteins, and activation of JNK1/2 and ERK1/2. Western blot analysis was performed after UVB treatment for $180 \mathrm{~min}$ to detect Bax, Bcl-2 and caspase-3 in HLECs. CALML3 downregulation markedly decreased the expression of the anti-apoptotic protein Bcl-2, and increased the expression of the pro-apoptotic proteins caspase- 3 and Bax compared with the NC group (Fig. 8A and B). Furthermore, CALML3 downregulation markedly suppressed the activation of JNK1/2 and ERK1/2 compared with the NC group (Fig. 8C and D) after
UVB treatment for 60 min of HLECs. However, nimodipine treatment significantly inhibited the effects of siCALML3 in HLECs. These findings indicate that JNK $1 / 2$ and ERK1/2 are involved in apoptosis induced by CALML3 downregulation in HLECs.

\section{Discussion}

Cataracts are a major cause of blindness and the only cure is surgical removal of the lens and replacement with a clear one. 
A

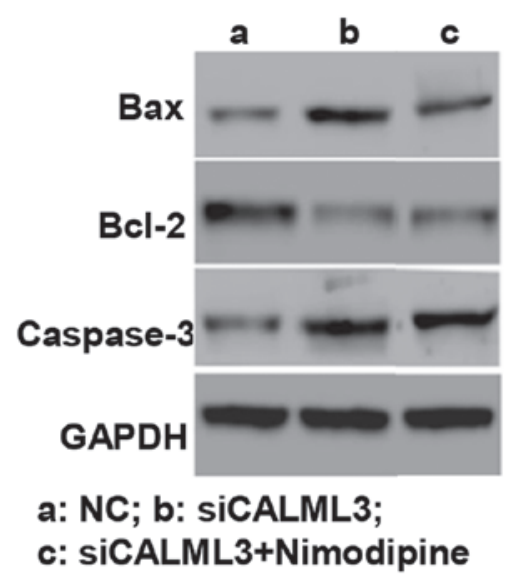

C

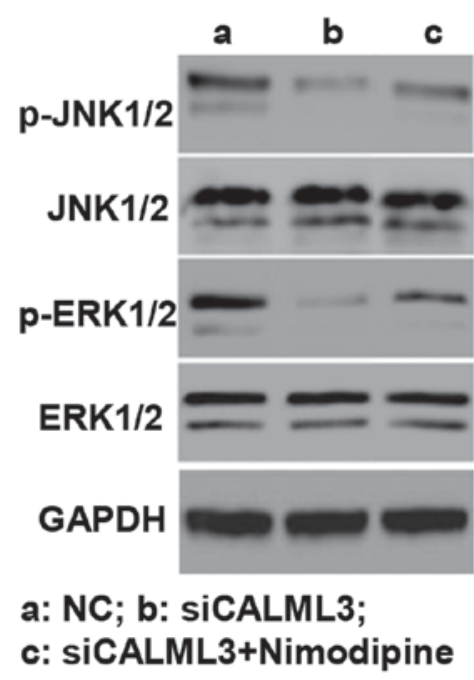

B

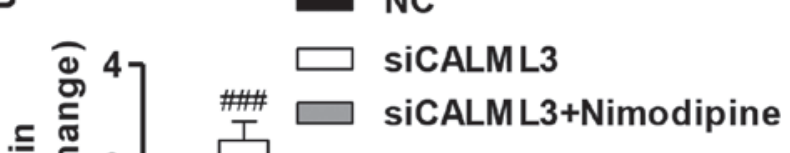

D

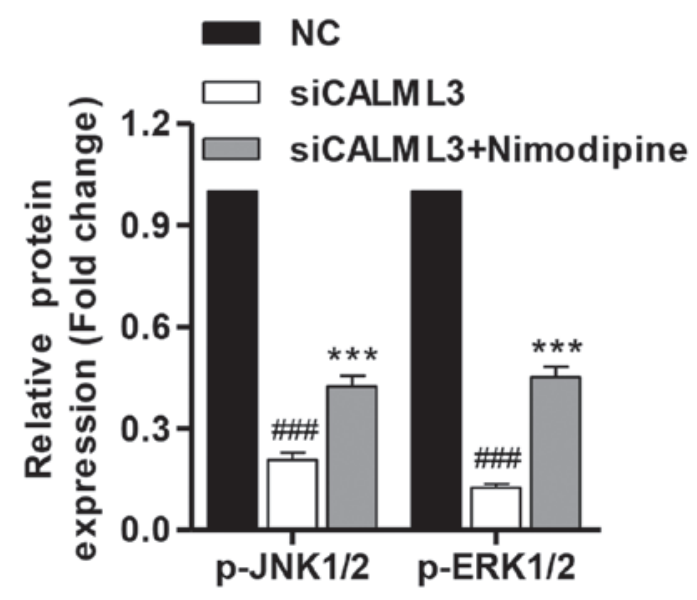

Figure 8. Effect of CALML3 downregulation on apoptosis associated proteins and JNK1/2 and ERK1/2 in HLECs. (A and B) The protein expression levels of caspase-3 and Bax/Bcl-2 ratio were significantly increased in HLECs with CALML3 downregulation in HLECs. (C and D) The phosphorylation levels of JNK1/2 and ERK1/2 were significantly decreased in HLECs with siCALML3 transfection. However, nimodipine treatment inhibited the effects of siCALML3-induced apoptotic proteins expression and JNK1/2 and ERK1/2 signaling pathways. ${ }^{\# \# / P}<0.001$ vs. NC and ${ }^{* * *} \mathrm{P}<0.001$ vs. siCALML3. NC, negative control; siCALML3, calmodulin like 3 small interfering RNA; p-, phospho-; JNK, c-Jun NH2-terminal kinase; ERK, extracellular signal-regulated kinase; HLECs, human lens epithelial cells;

HLECs are the center of metabolic activities in lens, and to prevent the onset of cataract is important determine the mechanisms of cataract development. UVB is a part of the sunlight spectrum and can cause retina damaged even $<1 \%$ of the UVB penetrates it. In the present study, the effects of UVB irradiation on apoptosis, ROS production and $\mathrm{Ca}^{2+}$ concentration of HLECs were examined using different intensities and duration of UVB exposure. Following different intensities and times of exposure to UVB irradiation, the number of apoptotic cells, ROS production and $\mathrm{Ca}^{2+}$ concentration was significantly increased in HLECs in both dose- and time-dependent manner. In agreement with our findings that UVB irradiation could induce HLECs apoptosis, produce ROS and cause $\mathrm{Ca}^{2+}$ elevation $(3,23)$.

Whether the mechanism of cataract induced apoptosis is involved in $\mathrm{Ca}^{2+}$ elevation has to be investigated. The current study examined the association of UVB irradiation with CALML3. After $2 \mathrm{~W} / \mathrm{m}^{2}$ UVB irradiation for 15,90 and $180 \mathrm{~min}$, the mRNA and protein levels of CALML3 were decreased in a time-dependent manner. The interesting pattern of expression indicated that may be a CALML3 a potentially attractive marker of cataract progression. Notably, overexpression of CALML3 in HLECs significantly reduced apoptosis,
ROS production and intracellular $\mathrm{Ca}^{2+}$ concentration following exposure of HLECs with $2 \mathrm{~W} / \mathrm{m}^{2} \mathrm{UVB}$ irradiation for $90 \mathrm{~min}$. Similar to the ubiquitous $\mathrm{Ca}^{2+}$ sensor protein calmodulin, CALML3 acts in pre-apoptotic cells by interacting with a nuclear protein involved in chromosome fragmentation (20). The findings of the current study suggest that UVB irradiation induces apoptosis by downregulating expression of CALML3, and upregulation of CALML3 can reverse the UVB-induced damage of HLECs.

Numerous genes are associated with apoptosis, such as the Bcl-2 family, Bax and caspase-3. Among the Bcl-2 family, Bcl-2 is an anti-apoptotic protein; however the Bax and caspase-3 are pro-apoptotic $(24,25)$. The current study measured the mRNA and protein expression levels of these proteins. The results demonstrated that overexpression of CALML3 significantly decreased the expression of Bax and caspase-3, and increased the expression of $\mathrm{Bcl}-2$, suggesting that upregulation of CALML3 reverses UVB-induced apoptosis by decreasing caspase- 3 and the $\mathrm{Bax} / \mathrm{Bcl}-2$ ratio. In agreement with these findings, Bax/Bcl-2 ratio and caspase-3 activity were highly positively correlated to the proportion of apoptotic cells in HLECs following UVB irradiation (7). 
Numerous scientific investigations have confirmed the presence of oxidative stress in ocular disease. Oxidative damage caused by ROS has an important role in the pathophysiology of many forms of cataract $(1,26)$. In order to protect against toxic effects of ROS, the cells have antioxidant defense systems, which consist of antioxidative enzymes including SOD, CAT and GSH-PX, and antioxidants such as MDA. Under normal conditions, HLECs maintain ROS at low levels via activation of SOD, CAT and GSH-PX, and MDA inhibition (27). However, some reported decreased SOD, CAT and GSH-PX, but increased MDA levels in cataracts (28), although other studies have reported conflicting results (29). In the present study, UVB-induced HLECs with CALML3 overexpression decreased the activities of SOD, CAT and GSH-PX, and increased the activity of MDA, compared with UVB-induced HLECs without CALML3 upregulation.

To further examine the association of CALML3 with cataracts, siRNA was used to knockdown CALML3 in HLECs. Silencing of CALML3 significantly increased the number of apoptotic cells, ROS production and intracellular $\mathrm{Ca}^{2+}$ concentration, which was similar to the effects of UVB in HLECs. However, nimodipine, a $\mathrm{Ca}^{2+}$-channel antagonist, inhibited ROS production and intracellular $\mathrm{Ca}^{2+}$ concentration, and had no effect on apoptosis. By contrast, other studies reported that nimodipine inhibited of apoptosis and ROS production (30) and is considered to be a potent antioxidant in vivo and in vitro $(31,32)$. In addition, nimodipine treatment partially rescued changes in expression of Bax, caspase-3 and Bcl-2 caused by CALML3 downregulation. These findings suggest that CALML3 downregulation induced apoptosis through activating the calcium signaling pathway.

It has been reported that the activation of the MAPK signaling cascade is essential for oxidative stress-induced apoptosis in HLECs (33), and the UVB-activated signal transduction pathways are predominantly mediated through signaling cascades involving MAPKs, including JNK, ERK and p38 kinases (23). Among these MAPKs, ERK is considered to be essential for cell survival (34), and JNK and p38 activation are considered to have a role in apoptotic signaling $(8,35)$. Herein, the association of CALML3 downregulation with apoptosis in HLECs was also investigated. The results demonstrated that downregulation of CALML3 significantly inhibited the activation of JNK1/2 and ERK1/2, whereas nimodipine treatment reversed the inhibition of JNK1/2 and ERK1/2 caused by CALML3A downregulation. Similar to these findings, the protein levels of p-JNK and p-ERK were negatively regulated by treatment with nimodipine in oxygen and glucose deprivation/reperfusion-induced cell injury in primary culture of rat cortical neurons (36). Thus, results of the present study provided evidence to suggest that JNK1/2 and ERK1/2 are involved in CALML3 downregulation and UVB-induced apoptosis.

Investigating the mechanism of apoptosis in HLECs may provide important insight into understanding the processes involved in cataract development. In the current study, UVB irradiation induced apoptosis, ROS production and intracellular $\mathrm{Ca}^{2+}$ concentration elevation in HLECs. Overexpression of CALML3 inhibited the damage caused by UVB irradiation and decreased the levels of caspase-3, Bax/Bcl-2 ratio and ROS production. In addition, downregulation of CALML3 induced HLEC apoptosis potentially through inhibition of
JNK1/2 and ERK1/2 activation. These results suggest that the UVB induces apoptosis in HLECs via oxidative stress, $\mathrm{Ca}^{2+}$, and JNK1/2 and ERK1/2 signaling pathways.

\section{Acknowledgements}

Not applicable

\section{Funding}

This study was supported by grants from the National 973 Foundation (no. 2013CB531606), the National Science Foundation of China (nos. 81300748, 81471605, 81273282, 81202353 and 81170834), the Shanghai Shenkang Grant (no. CHDC22014014), the Grant of the Army Scientific Research (no. BWS14J023).

\section{Availability of data and materials}

All data generated or analyzed during this study are included in this published article.

\section{Authors' contributions}

YJ and QQ conceived and designed the experiments. CPF, WS and TTS performed the experiments. YLH analyzed the data. WJL and AMD contributed as regards the reagents/materials/ analysis tools. YJ, WJL and AMD wrote the paper. All authors read and approved the final manuscript.

\section{Ethics approval and consent to participate}

Not applicable

\section{Consent for publication}

Not applicable

\section{Competing interests}

The authors declare that they have no competing interests.

\section{References}

1. Shibata S, Singh DP, Shibata N, Sasaki H and Kubo E: Hyperglycemia-and oxidative stress-induced hyperoxidation of Prdx 6 during lens epithelial cell damage and rat lens opacity in vitro and in vivo. Invest Ophthalmol Vis Sci 55: 5723-5723, 2014.

2. Giblin FJ, Lin L-R, Leverenz VR and Dang L: A class I (Senofilcon A) soft contact lens prevents UVB-induced ocular effects, including cataract, in the rabbit in vivo. Invest Ophthalmol Vis Sci 52: 3667-3675, 2011.

3. Wu Q, Guo D, Bi H, Wang D and Du Y: UVB irradiation-induced dysregulation of plasma membrane calcium ATPasel and intracellular calcium homeostasis in human lens epithelial cells. Mol Cell Biochem 382: 263-272, 2013.

4. Zhang J, Yan H, Löfgren S, Tian X and Lou MF: Ultraviolet radiation-induced cataract in mice: The effect of age and the potential biochemical mechanism. Invest Ophthalmol Vis Sci 53: 7276-7285, 2012.

5. Kim J, Kim OS, Kim C-S, Sohn E, Jo K and Kim JS: Accumulation of argpyrimidine, a methylglyoxal-derived advanced glycation end product, increases apoptosis of lens epithelial cells both in vitro and in vivo. Exp Mol Med 44: 167-175, 2012.

6. Sinha K, Das J, Pal PB and Sil PC: Oxidative stress: The mitochondria-dependent and mitochondria-independent pathways of apoptosis. Arch Toxicol 87: 1157-1180, 2013. 
7. Ji Y, Cai L, Zheng T, Ye H, Rong X, Rao J and Lu Y: The mechanism of UVB irradiation induced-apoptosis in cataract. Mol Cell Biochem 401: 87-95, 2015.

8. Zwang Y and Yarden Y: p38 MAP kinase mediates stress-induced internalization of EGFR: Implications for cancer chemotherapy. EMBO J 25: 4195-4206, 2006.

9. Roduit $\mathrm{R}$ and Schorderet DF: MAP kinase pathways in UV-induced apoptosis of retinal pigment epithelium ARPE19 cells. Apoptosis 13: 343-353, 2008.

10. Chang D, Zhang X, Rong S, Sha Q, Liu P, Han T and Pan H: Serum antioxidative enzymes levels and oxidative stress products in age-related cataract patients. Oxid Med Cell Longev 2013: 587826, 2013.

11. Wojcik M, Burzynska-Pedziwiatr I and Wozniak LA: A review of natural and synthetic antioxidants important for health and longevity. Curr Med Chem 17: 3262-3288, 2010.

12. Yonar ME: The effect of lycopene on oxytetracycline-induced oxidative stress and immunosuppression in rainbow trout (Oncorhynchus mykiss, W.). Fish Shellfish Immunol 32: 994-1001, 2012.

13. Dechandt CR, De Souza DL, Siqueira JT, Pereira MP, De Assis RP, Da Silva VC, De Sousa PT Jr, Brunetti IL, Andrade CM, Kawashita $\mathrm{NH}$, et al: Changes in the oxidative stress biomarkers in liver of streptozotocin-diabetic rats treated with combretum lanceolatum flowers extract. Br J Pharm Res 4: 2340-2356, 2014.

14. Chang D, Zhang X, Rong S, Sha Q, Liu P, Han T and Pan H: Serum antioxidative enzymes levels and oxidative stress products in age-related cataract patients. Oxid Med Cell Longev 2013: 587826, 2013.

15. Strehler EE: Emanuel Strehler's work on calcium pumps and calcium signaling. World J Biol Chem 2: 67-72, 2011.

16. Chang KC, Lee AS, Chen WY, Lin YN, Hsu JF, Chan HC Chang CM, Chang SS, Pan CC, Sawamura T, et al: Increased LDL electronegativity in chronic kidney disease disrupts calcium homeostasis resulting in cardiac dysfunction. J Mol Cell Cardiol 84: 36-44, 2015.

17. Fu S, Yang L, Li P, Hofmann O, Dicker L, Hide W, Lin X Watkins SM, Ivanov AR and Hotamisligil GS: Aberrant lipid metabolism disrupts calcium homeostasis causing liver endoplasmic reticulum stress in obesity. Nature 473: 528-531, 2011.

18. Walton JR: Aluminum disruption of calcium homeostasis and signal transduction resembles change that occurs in aging and Alzheimer's disease. J Alzheimers Dis 29: 255-273, 2012.

19. Brooks MD, Bennett RD, Weaver AL, Sebo TJ, Eckert SE, Strehler EE and Carr AB: Human calmodulin-like protein CALML3: a novel marker for normal oral squamous mucosa that is downregulated in malignant transformation. Int J Dent 2013 592843, 2013.

20. Bennett RD, Pittelkow MR and Strehler EE: Immunolocalization of the tumor-sensitive calmodulin-like protein CALML3 in normal human skin and hyperproliferative skin disorders. PLoS One 8: e62347, 2013.

21. Calenda G, Suadicani SO, Iglesias R, Spray DC, Melman A and Davies KP: Silencing MaxiK activity in corporal smooth muscle cells initiates compensatory mechanisms to maintain calcium homeostasis. J Sex Med 8: 2191-2204, 2011.
22. Livak and Schmittgen: Analysis of relative gene expression data using real-time quantitative PCR and the $2-\Delta \Delta \mathrm{Ct}$ method. Methods 25: 402-408, 2001.

23. Cao G, Chen M, Song Q, Liu Y, Xie L, Han Y, Liu Z, Ji Y and Jiang Q: EGCG protects against UVB-induced apoptosis via oxidative stress and the JNK1/c-Jun pathway in ARPE19 cells. Mol Med Rep 5: 54-59, 2012.

24. Tsukahara S, Yamamoto S, Tin-Tin-Win-Shwe, Ahmed S, Kunugita N, Arashidani K and Fujimaki $\mathrm{H}$ : Inhalation of low-level formaldehyde increases the $\mathrm{Bcl}-2 / \mathrm{Bax}$ expression ratio in the hippocampus of immunologically sensitized mice. Neuroimmunomodulation 13: 63-68, 2006.

25. Xu GX and Wang TT: Apoptosis of lens epithelial cells induced by cinobufagin in vitro. Int J Ophthalmol 3: 128-131, 2010.

26. Klein B, Myers C, Cruickshanks K, Lee K and Klein R: Inflammatory and oxidative stress markers and the 20-year cumulative incidence of age-related cataract: The beaver dam eye study. Invest Ophthalmol Vis Sci 54: 897-897, 2013.

27. Berthoud VM and Beyer EC: Oxidative stress, lens gap junctions, and cataracts. Antioxid Redox Signal 11: 339-353, 2009.

28. Brennan LA and Kantorow M: Mitochondrial function and redox control in the aging eye: Role of MsrA and other repair systems in cataract and macular degenerations. Exp Eye Res 88: 195-203, 2009.

29. Scharf J and Dovrat A: Superoxide dismutase molecules in human cataractous lenses. Ophthalmic Res 18: 332-337, 1986.

30. Wang D, Wang Z, Li Y, Chen X and Sun GY: Nimodipine inhibits $\mathrm{N}$-methyl-N-nitrosourea-induced retinal photoreceptor apoptosis in vivo. Indian J Pharmacol 45: 149-154, 2013.

31. Ambhore N, Prasanna M, Antony A, Satish Kumar M and Elango K: Pharmacological and anti-oxidant evaluation of Aspirin, nimodipine and its combination for anti-Parkinson's activity in MPTP induced rat model. Int J Health Allied Sci 3: 14, 2014.

32. Yu L, Wang N, Zhang Y, Wang Y, Li J, Wu Q and Liu Y: Neuroprotective effect of muscone on glutamate-induced apoptosis in $\mathrm{PC} 12$ cells via antioxidant and $\mathrm{Ca}(2+)$ antagonism. Neurochem Int 70: 10-21, 2014.

33. Amin KK and Shah A: Prevention of oxidative stress and cell death in human lens epithelial cells by guggulsterone. Invest Ophthalmol Vis Sci 53: 1070-1070, 2012.

34. Dent P, Yacoub A, Fisher PB, Hagan MP and Grant S: MAPK pathways in radiation responses. Oncogene 22: 5885-5896, 2003.

35. Li T, Dai W and Lu L: Ultraviolet-induced junD activation and apoptosis in myeloblastic leukemia ML-1 cells. J Biol Chem 277: 32668-32676, 2002.

36. Wang CP, Li GC, Shi YW, Zhang XC, Li JL, Wang ZW, Ding F and Liang XM: Neuroprotective effect of schizandrin A on oxygen and glucose deprivation/reperfusion-induced cell injury in primary culture of rat cortical neurons. J Physiol Biochem 70: 735-747, 2014. 